\title{
SEMICLASSICAL MOTION OF SYSTEMS WITH INTERNAL STATES
}

\begin{abstract}
S. Stenimolm
Research Institute for Theoretical Physics and the Academy of Finland P.O. Box 9 (Siltavuorenpenger 20C), 00014 University of Ilelsinki, Finland

In many fields of atomic and molecular physics we need to rom! in il. center-of-mass motion with internal degrees of freedom. In this work, the classical motion in phase space is separated from the quantum processes by a multiple-time-scale approach. The cxpansion parameter is proportional to Planck's constant. The theory is given and illustrated by examples: the semiclassical description of a particle without internal states, the extraction of a classical trajectory and the derivation of the light-induced force.
\end{abstract}

PACS numbers: 03.65.Sq, 32.80.Pj

\section{Introduction}

The relationship between the quantum behaviour of a physical system and its classical trajectories has turned out to be more complicated and interesting than the text book semiclassical approximation suggests. Eric IIeller [1] terms this field "postmodern quantum mechanics", and its recent technical developments have been reviewed by Child in [2]. The connection to chaos theory is discussed by de Almeida in [3]. The relation between semiclassical distributions in phase space and classical ensembles is also discussed by Dowling et al. in [4]. All these treatments do, however, use steady state wave functions corresponding to fixed energy ensembles. In time resolved experiments, one wants to develop the theory for genuinely time dependent problems too.

In many atomic physics and quantum chemistry situations the quantum systems evolving in phase space have internal states that correspond to bound states. These are coupled by collisions or external electromagnetic fields. Thus it becomes interesting to discuss the semiclassical time evolution of a system with internal states. In the recent publication [5] we have utilized the multiple-time-scale perturbation approach [6] to separate different processes according to their dependence on Planck's constant. Heller [7] has pointed out that the initial states, e.g. minimum uncertainty states, add dependence on Planck's constant which makes such an expansion ambiguous. We avoid this problem by separating the processes 
into a lierarchy of classes. This is achieved by expanding the propagators for an arbitrary initial state, which can be done uniquely. For a discussion of the procedure and its relation to earlier work, we refer to the paper [5].

Here we introduce the expansion method in Sec. 2. By omitting the internal states from the treatment, in Sec. 3, we can connect our results with the constant energy ensembles discussed earlier. In Sec. 4 a classical trajectory approximation is derived; this corresponds to the pre-assigned trajectory approximation in scattering theory and level crossing theories. The light-induced mechanical force is derived in Sec. 5, where the results known from e.g. Ref. [8] are obtained. In Sec. 6 a brief discussion is presented.

\section{The multiple time-scale expansion}

The Wigner function is assumed to be particularly useful when the semiclassical content of quantum mechanics is desired [9]. The Wigner transformed density matrix $\rho$ is given by

$$
W(r, p)=\frac{1}{(2 \pi \hbar)^{d}} \int \exp (-\mathrm{i} p x / \hbar)\left\langle r+\frac{1}{2} x|\rho| r-\frac{1}{2} x\right\rangle \mathrm{d}^{d} x .
$$

Here the state $|r\rangle$ is the position eigenstate and vector notation is understood.

The transformation (1) to the quantum mechanical phase space $\{r, p\}$ concerns only the translational degrees of freedom of the quantum system; we assume that this has an additional set of intrinsic degrees of freedom manifesting themselves as a set of discrete bound states. If we truncate the corresponding Hilbert space to be of finite dimensions, the density operator becomes a matrix $\rho_{i j}$ with indices labelling the internal states. Then the Wigner representation of the density matrix retains its operator structure in the space of the internal states.

The equation of motion for the Wigner function can be written in the form

$$
\begin{aligned}
& \frac{\partial}{\partial t} W(r, p)+\frac{p}{M} \frac{\partial}{\partial r} W(r, p) \\
& \quad=\frac{1}{\mathrm{i} \hbar}\left[V\left(r+\frac{\mathrm{i} \hbar}{2} \frac{\vec{\partial}}{\partial p}\right) W(r, p)-W(r, p) V\left(r-\frac{\mathrm{i} \hbar}{2} \frac{\overleftarrow{\partial}}{\partial p}\right)\right] .
\end{aligned}
$$

The potential $V$ is a matrix in the internal indices and the derivatives act in the direction indicated by the arrow.

The semiclassical expansion is well known to proceed in powers of Planck's constant $\hbar$. This is not a dimensionless expansion parameter, and we return to this problem in Sec. 6. We expand (2) and obtain the result

$$
\begin{aligned}
\frac{\partial}{\partial t} W & +\frac{p}{M} \frac{\partial}{\partial r} W=\frac{1}{\mathrm{i} h}[V, W]+\frac{1}{2}\left[\frac{\partial V}{\partial r}, \frac{\partial W}{\partial p}\right]_{+} \\
+ & \frac{\mathrm{i} h}{8}\left[\frac{\partial^{2} V}{\partial r^{2}}, \frac{\partial^{2} W}{\partial p^{2}}\right]-\frac{\hbar^{2}}{48}\left[\frac{\partial^{3} V}{\partial r^{3}}, \frac{\partial^{3} W}{\partial p^{3}}\right]_{+}+\mathrm{O}\left(\hbar^{3}\right) .
\end{aligned}
$$

IIere $[,]_{+}$denotes the anticommutator. This expansion is clearly in powers of $\hbar$, and for no internal states all commutator terms vanish; this reproduces the conventional semiclassical expansion of the Wigner function. 
We expect the quantum mechanical and classical features of the time evolution to be observable over different time scales. From the ordinary semiclassical expansion we conclude that the pure quantum time scale is of the order $\hbar^{-1}$, and consequently we introduce a sequence of time scales by the definition

$$
T_{\nu}=h^{\nu-1} t, \quad \nu=0,1,2, \ldots
$$

The time derivative becomes (see Ref. [6])

$$
\frac{\partial}{\partial t}=\frac{1}{\hbar} \frac{\partial}{\partial T_{0}}+\frac{\partial}{\partial T_{1}}+\hbar \frac{\partial}{\partial T_{2}}+\hbar^{2} \frac{\partial}{\partial T_{3}}+\ldots
$$

and the density matrix is expanded as a simple power series in $\hbar$ according to

$$
W=W^{(0)}+\hbar W^{(1)}+\hbar^{2} W^{(2)}+\hbar^{3} W^{(3)}+\ldots
$$

When the expansions (5) and (6) are inserted into Eq. (3) and equal powers of $\hbar$ are collected, we obtain the hierarchy of equations:

Order $\hbar^{-1}$

$$
\frac{\partial W^{(0)}}{\partial T_{0}}-\frac{1}{\mathrm{i}}\left[V(r), W^{(0)}\right]=0
$$

Order $\hbar^{0}$

$$
\frac{\partial W^{(1)}}{\partial T_{0}}-\frac{1}{\mathrm{i}}\left[V(r), W^{(1)}\right]=-\left\{\frac{\partial W^{(0)}}{\partial T_{1}}+\frac{p}{M} \frac{\partial W^{(0)}}{\partial r}-\frac{1}{2}\left[\frac{\partial V}{\partial r}, \frac{\partial W^{(0)}}{\partial p}\right]_{+}\right\},
$$

Order $\hbar^{1}$

Order $\hbar^{2}$

$$
\begin{aligned}
\frac{\partial W^{(2)}}{\partial T_{0}}- & \frac{1}{\mathrm{i}}\left[V(r)_{i} W^{(2)}\right]=-\left\{\frac{\partial W^{(1)}}{\partial T_{1}}+\frac{p}{M} \frac{\partial W^{(1)}}{\partial r}-\frac{1}{2}\left[\frac{\partial V}{\partial r}, \frac{\partial W^{(1)}}{\partial p}\right]_{+}\right\} \\
& -\left\{\frac{\partial W^{(0)}}{\partial T_{2}}-\frac{\mathrm{i}}{8}\left[\frac{\partial^{2} V}{\partial r^{2}}, \frac{\partial^{2} W^{(0)}}{\partial p^{2}}\right]\right\},
\end{aligned}
$$

$$
\begin{aligned}
& \frac{\partial W^{(3)}}{\partial T_{0}}-\frac{1}{\mathrm{i}}\left[V(r), W^{(3)}\right]=-\left\{\frac{\partial W^{(2)}}{\partial T_{1}}+\frac{p}{M} \frac{\partial W^{(2)}}{\partial r}-\frac{1}{2}\left[\frac{\partial V}{\partial r}, \frac{\partial W^{(2)}}{\partial p}\right]_{+}\right\} \\
& -\left\{\frac{\partial W^{(1)}}{\partial T_{2}}-\frac{\mathrm{i}}{8}\left[\frac{\partial^{2} V}{\partial r^{2}}, \frac{\partial^{2} W^{(1)}}{\partial p^{2}}\right]\right\}-\left\{\frac{\partial W^{(0)}}{\partial T_{3}}+\frac{1}{48}\left[\frac{\partial^{3} V}{\partial r^{3}}, \frac{\partial^{3} W^{(0)}}{\partial p^{3^{3}}}\right]_{+}\right\} .(10)
\end{aligned}
$$

To lowest order in $\hbar$, Eq. (7) expresses the quantum time evolution at the location $r$; reintroducing $\hbar$, we find the correct Schrödinger time dependence. To order $\hbar^{0}$, Eq. (8) contains the classical drift derivative in phase space. The anticommutator contains the force matrix, which in the case of a diagonal potential gives the classical Liouville equation on the potential surfaces. However, the operator on the left-hand side needs to be inverted in order to give the new unknown $W^{(1)}$, and this operator is identical with the one in Eq. (7). Thus the inhomogeneous term on the right-hand side of (8) is in the null space of this operator, and the inversion may not be possible. This is a situation familiar from all singular perturbation theory problems. In the multiple-time-scale method, we use the additional degrees of freedom offered by the many times to circumvent this difficulty. 
The following terms in the expansion, e.g. (9) and (10) follow the same pattern. At each stage the new function to be determined resides inside the operator inverted to obtain the previous ones, and time evolution with respect to $T_{1}(=t)$ is always given by the classical Liouville operator. The structure of the equations in each order is a replica of the one in the previous order but with one additional term introducing a new operator. We will now proceed to investigate some simple cases and their consequences.

\section{The scalar case}

To establish contact with some earlier work, we consider the scalar situation where no internal degrees of freedom occur. Then all commutator terms vanish and we are left with the equations

$$
\begin{aligned}
& \frac{\partial W^{(0)}}{\partial T_{0}}=0 \\
& \frac{\partial W^{(1)}}{\partial T_{0}}=-\left\{\frac{\partial W^{(0)}}{\partial T_{1}}+\frac{p}{M} \frac{\partial W^{(0)}}{\partial r}-\frac{\partial V}{\partial r} \frac{\partial W^{(0)}}{\partial p}\right\} \\
& \frac{\partial W^{(2)}}{\partial T_{0}}=-\left\{\frac{\partial W^{(1)}}{\partial T_{1}}+\frac{p}{M} \frac{\partial W^{(1)}}{\partial r}-\frac{\partial V}{\partial r} \frac{\partial W^{(1)}}{\partial p}\right\}-\frac{\partial W^{(0)}}{\partial T_{2}} \\
& \frac{\partial W^{(3)}}{\partial T_{0}}=-\left\{\frac{\partial W^{(2)}}{\partial T_{1}}+\frac{p}{M} \frac{\partial W^{(2)}}{\partial r}-\frac{\partial V}{\partial r} \frac{\partial W^{(2)}}{\partial p}\right\} \\
& -\frac{\partial W^{(1)}}{\partial T_{2}}-\left\{\frac{\partial W^{(0)}}{\partial T_{3}}+\frac{1}{24} \frac{\partial^{3} V}{\partial r^{3}} \frac{\partial^{3} W^{(0)}}{\partial p^{3}}\right\}
\end{aligned}
$$

A way to solve these equations is to set all even order lime derivatives equal to zero, in particular no quantum evolution is included, viz.

$$
\frac{\partial W}{\partial T_{0}}=\frac{\partial W}{\partial T_{2}}=0
$$

The dependence on the time $T_{1}$ is determined such that we satisfy the classical Liouville equation

$$
\frac{\partial W}{\partial T_{1}}+\frac{p}{M} \frac{\partial W}{\partial r}-\frac{\partial V}{\partial r} \frac{\partial W}{\partial p}=0
$$

in all orders. This equation is solved by the construction of the classical trajectories obeying the characteristic equations

$$
\frac{\mathrm{d} r}{\mathrm{~d} T_{1}}=\frac{p}{M}, \quad \frac{\mathrm{d} p}{\mathrm{~d} T_{1}}=-\frac{\partial V}{\partial r}
$$

with the initial conditions

$$
r=r_{0}, \quad p=p_{0} \text { for } T_{1}=0 .
$$

Denoting these solutions by $r\left(T_{1} \mid r_{0}, p_{0}\right)$ and $p\left(T_{1} \mid r_{0}, p_{0}\right)$, we find the solution

$$
W_{0}\left(r, p, T_{1} \mid r_{0}, p_{0}\right)=\delta\left(r-r\left(T_{1} \mid r_{0}, p_{0}\right)\right) \delta\left(p-p\left(T_{1} \mid r_{0}, p_{0}\right)\right)
$$


for a single trajectory. Multiplied by any initial distribution $P_{0}\left(r_{0}, p_{0}\right)$ this gives the general solution to Eq. (16). The dependence on the time $T_{3}$ is, however, not determined, but this can be fixed using Eq. (14) in the form

$$
\frac{\partial W^{(0)}}{\partial T_{3}}=-\frac{1}{24} \frac{\partial^{3} V}{\partial r^{3}} \frac{\partial^{3} W^{(0)}}{\partial p^{3}}
$$

This may be used to define the dependence of the solution $W^{(0)}$ on the momentum variable $p$, but the space dependence remains free, let us retain the $\delta$-function for this.

We assume that the derivative $\partial^{3} V / \partial r^{3}$ can be replaced by a constant $V^{\prime \prime \prime}$; either the potential has no higher derivatives or we assume a local expansion of the potential around the trajectory (16)-(19) in the spirit of Heller [10]. When $\hbar$ is reintroduced we obtain the solution to Eq. (20) in the form

$$
W^{(0)}\left(r, p, t \mid r_{0}, p_{0}\right)=C A \mathrm{~A}\left[\frac{2\left(p-p\left(t \mid r_{0}, p_{0}\right)\right)}{\hbar^{2 / 3} t^{1 / 3}\left|V^{\prime \prime \prime}\right|^{1 / 3}}\right] \delta\left(r-r\left(t \mid r_{0}, p_{0}\right)\right)
$$

where we have introduced the Airy function

$$
\operatorname{Ai}(x / a)=\frac{a}{2 \pi} \int_{-\infty}^{+\infty} \exp \left(\mathrm{i} t x+\mathrm{i} \frac{1}{3} t^{3} a^{3}\right) \mathrm{d} t \Rightarrow a \delta(x) \text { for } a \Rightarrow 0 .
$$

The normalization requires that

$$
\int_{-\infty}^{+\infty} W^{(0)}\left(r, p, t \mid r_{0}, p_{0}\right) \mathrm{d} r \mathrm{~d} p=1
$$

which from Eqs. (21) and (22) fixes the prefactor

$$
C=\frac{2}{\hbar^{2 / 3} t^{1 / 3}\left|V^{\prime \prime \prime}\right|^{1 / 3}}
$$

In order to connect our approximate Wigner function (21) to other semiclassical expansions we look at a simple case of one-dimensional periodic motion. In this case a unique relation exists between time and position along the trajectory for each half period. We choose $p_{0}=0$ at time $t=0$, and start the evolution. The energy of the trajectory is determined by the relation

$$
E=\frac{p^{2}}{2 M}+V(r)=V\left(r_{0}\right) .
$$

As we move along the trajectory, this conserved quantity serves to define the relation between $r$ and $p$. However, the dependence on $r_{0}$ can now effectively be replaced by the dependence on energy and hence we can write

$$
p\left(t \mid r_{0}, p_{0}=0\right)=p(r(t), E),
$$

because there is a one-to-one correspondence between time and the position along the given trajectory according to Eq. (25).

In order to obtain the Wigner function for a microcanonical distribution we collect an ensemble consisting of all positions along the given trajectory by averaging over the half period $T_{0} / 2$. We find 


$$
\begin{aligned}
& \frac{2}{T_{0}} \int_{0}^{T_{0} / 2} \mathrm{~d} l \delta\left(r-r\left(t \mid r_{0}, p_{0}\right)\right) \\
& \quad=\frac{2}{T_{0}} \int_{0}^{T_{0} / 2} \mathrm{~d} t \frac{\delta\left(t-t\left(r \mid r_{0}, p_{0}\right)\right)}{v\left(t \mid r_{0}, p_{0}\right)}=\frac{2 M}{T_{0} p(r(t), E)},
\end{aligned}
$$

where the time now denotes

$$
t=t\left(r \mid r_{0}, p_{0}\right)=t(r, E) .
$$

This transforms the function $r(t)$ in Eqs. (26) and (28) into the variable $r$, and $t$ is replaced according to (28) everywhere.

The constant energy Wigner function now becomes the microcanonical distribution

$$
W(r, p \mid E)=\frac{2 M}{T_{0} p(r, E)} C \mathrm{Ai}[C(p-p(r \mid E))],
$$

where $p(r, E)$ is solved from (25) and the period is given by its classical expression

$$
T_{0}=2 M \int_{r_{0}}^{r_{1}} \frac{\mathrm{d} r}{p(r \mid E)} \text {. }
$$

The relation (29) indicates correctly that the relative weight of each position along the trajectory is inversely proportional to the velocity, as expected from simple classical considerations. In the limit $C \Rightarrow \infty$, we obtain with Eq. (22) the result

$$
W(r, p \mid E) \Rightarrow \frac{2 M}{T_{0} p(r, E)} \delta(p-p(r \mid E))
$$

using (30) it is easy to see that this expression is correctly normalized. To see the meaning of the relation (31) we write

$$
\delta(p-p(r \mid E))=\frac{1}{2}\left(\frac{\partial E}{\partial p}\right) \delta(E-E(r, p)),
$$

where $E(r, p)$ is given by Eq. (25) and the factor 2 derives from the two values of $p$ corresponding to each given pair $\{r, E\}$. Inserting into (31) we find the microcanonical distribution

$$
W(r, p \mid E)=\frac{1}{T_{0}} \delta(E-E(r, p)) .
$$

In order to see that this is correctly normalized, we transform the variable $E$ to the corresponding action variable $I$ defined by

$$
E=\frac{2 \pi}{T_{0}} I .
$$

From (33) we find the distribution function in the action-angle phase plane $\{I, \varphi\}$ in the form

$$
W(I, \varphi)=\frac{1}{2 \pi} \delta(I-I(r, p)) .
$$

This is trivially seen to be correctly normalized, and it also agrees with the result derived by de Almeida (see Ref. [3], p. 173). Our procedure differs, however, from the one used to derive semiclassical Wigner functions from JWKB wave functions. As we have seen, for the microcanonical ensemble, the two approaches agree exactly. 


\section{The case of average motion}

We assume now that the potential part of the IIamiltonian can be divided into an average scalar potential $U(r)$ and a slowly varying remainder $\Delta V(r)$ viz.

$$
V(r)=U(r)+\Delta V(r)
$$

The quantum equation (7) immediately gives

$$
\frac{\partial W^{(0)}}{\partial T_{0}}=\frac{1}{\mathrm{i}}\left[\Delta V(r), W^{(0)}\right]
$$

which implies an optical Bloch equation at each point in space:

The next equation (8) in the hierarchy gives

$$
\frac{\partial W^{(1)}}{\partial T_{0}}-\frac{1}{\mathrm{i}}\left[\Delta V(r), W^{(1)}\right]=-\left\{\frac{\partial W^{(0)}}{\partial T_{1}}+\frac{p}{M} \frac{\partial W^{(0)}}{\partial r}-\frac{\partial U}{\partial r} \frac{\partial W^{(0)}}{\partial p}\right\} \text {. }
$$

In writing this equation we have neglected the slowly varying contribution

$$
Q_{1}=\frac{1}{2}\left[\frac{\partial \Delta V}{\partial r}, \frac{\partial W^{(0)}}{\partial p}\right]_{+} .
$$

If we again use the freedom offered by the multiple-time scales, we can solve Eq. (38) for $W^{(1)}$ by letting $W^{(0)}$ depend on $T_{1}$ in such a way that the right hand side of (38) disappears. This gives a classical Liouville equation which can be solved as we solved (16) in the previous section. Selecting one single trajectory by setting the initial conditions as in Eq. (18) we find in analogy with (19) the solution

$$
W_{0}\left(r, p, T_{1}, T_{0} \mid r_{0}, p_{0}\right)=\sigma\left(T_{0}\right) \delta\left(r-r\left(T_{1} \mid r_{0}, p_{0}\right)\right) \delta\left(p-p\left(T_{1} \mid r_{0}, p_{0}\right)\right)
$$

where $\sigma$ is a $2 \times 2$ density matrix depending on $T_{0}$. Inserting the solution (40) into Eq. (37) and integrating over the phase space $\{r, p\}$ we find the equation

$$
\mathrm{i} \hbar \frac{\partial \sigma}{\partial t}=\left[\Delta V\left(r\left(t \mid r_{0}, p_{0}\right)\right), \sigma\right]
$$

where we have reintroduced Planck's constant. We find the Bloch equation we would use with a pre-assigned trajectory determined by the initial conditions $\left\{r_{0}, p_{0}\right\}$ at $t=0$. This is exactly the procedure we use in the ordinary semiclassical approximation for atomic motion in quantum optics and scattering theory.

In next order we find the new equation

$$
\mathrm{i} \frac{\partial W^{(0)}}{\partial T_{2}}=-\frac{1}{8}\left[\frac{\partial^{2} \Delta V}{\partial r^{2}}, \frac{\partial^{2} W^{(0)}}{\partial p^{2}}\right] .
$$

Using the ansatz (40) we find directly

$$
\frac{\partial}{\partial T_{2}} \text { Trace } \sigma=0 \text {. }
$$

If Trace $\sigma$ is a constant, no dependence on $T_{2}$ needs to be introduced.

\section{The mechanical effect of light}

In this section we are going to consider a case where the spatial dependence of the IIamiltonian derives from the dipole interaction with an external light field $E(r, t)$. In the case of a two-level atom the interaction IIamiltonian becomes

$$
H_{\text {int }}=-\left[\begin{array}{ll}
0 & \mu \\
\mu & 0
\end{array}\right] E(r, t) \text {. }
$$


To the lowest order equation (7) we now add some linear relaxation terms to obtain the equation

$$
\mathrm{i} \frac{\partial W^{(0)}}{\partial T_{0}} \doteq\left[H_{\text {int }}(r), W^{(0)}\right]-\mathcal{R}\left(W^{(0)}\right) .
$$

In the rotating-wave approximation, this equation can be solved in steady state and we can try a solution in terms of the ansatz

$$
W^{(0)}=\sigma w^{0},
$$

where $w^{0}$ is a classical distribution function and $\sigma$ is a $2 \times 2$ density matrix satisfying

$$
\text { Trace } \sigma=1 \text {. }
$$

The result (8) gives the equation

$$
\frac{\partial W^{(0)}}{\partial T_{1}}+\frac{p}{M} \frac{\partial W^{(0)}}{\partial r}-\frac{1}{2}\left[\frac{\partial}{\partial r} H_{\mathrm{int}}, \frac{\partial W^{(0)}}{\partial p}\right]_{+}=0 .
$$

Taking the trace of this, we find for the distribution function $w^{0}$ the result

$$
\frac{\partial w^{0}}{\partial t}+\frac{p}{M} \frac{\partial w^{0}}{\partial r}+F \frac{\partial w^{0}}{\partial p}=0
$$

where we have introduced the physical time variable $t$ and the effective force

$$
F=-\operatorname{Tr}\left\{\frac{1}{2}\left[\frac{\partial}{\partial r} H_{\mathrm{int}}, \sigma\right]_{+}\right\}=\mu \frac{\partial E(r, t)}{\partial r}\left(\sigma_{21}+\sigma_{12}\right) .
$$

The elements of the density matrix $\sigma$ are to be determined from the steady state solution of Eq. (45).

If the radiation field is of the form

$$
E(r, t)=A(r) \exp [\mathrm{i}(\Omega t+\varphi(r))]+\text { c.c. }
$$

the off-diagonal elements of the density matrix can be written in the form

$$
\sigma_{21}=A(r) \chi \exp [\mathrm{i}(\Omega \iota+\varphi(r))],
$$

where $\chi$ is the two-level susceptibility which, for the case of spontaneous emission between the levels at the rate $\gamma$, can be written

$$
\chi=\frac{\mu}{2 h} \frac{\Delta+\mathrm{i} \gamma}{\Delta^{2}+\gamma^{2}(1+I)},
$$

where $\Delta=\omega_{21}-\Omega$ is the detuning and the saturation parameter is

$$
I=\frac{\mu^{2} A^{2}}{\hbar^{2} \gamma^{2}} \text {. }
$$

Inserting (51) and (52) into (50) and using the rotating-wave approximation, we find the force

$$
F=\mu \frac{\partial A^{2}}{\partial r} \operatorname{Re} \chi+2 \frac{\partial \varphi}{\partial r} \mu A^{2} \operatorname{Im} \chi .
$$

This shows both a dissipative and a reactive component to the light-induced force. For a plane wave, the phase is $\varphi=k r$, and we obtain the well-known expression

$$
F_{\text {diss }}=\hbar k \gamma \frac{I \gamma^{2}}{\Delta^{2}+\gamma^{2}(1+I)}
$$


For large laser intensities $I$, this gives the correct saturated expression for the light force $[8]$.

The reactive component is given by

$$
F_{\text {react }}=\frac{\hbar \Delta}{2} \frac{\partial I}{\partial r} \frac{\gamma^{2}}{\Delta^{2}+\gamma^{2}(1+I)}=-\frac{\partial \mathcal{U}(r)}{\partial r} ;
$$

in contrast to the light pressure force, this gradient force is seen to follow from the potential

$$
\mathcal{U}(r)=-\frac{\hbar \Delta}{2} \log \left(\Delta^{2}+\gamma^{2}(1+I)\right) .
$$

If the laser is tuned below the atomic resonance, $\Omega<\omega_{21}$, the two-level system is drawn towards regions with larger light intensity. This effect has been used to construct pure light traps for neutral atoms [8].

Considering the higher order corrections, Eq. (9), we can include quantum fluctuations into our treatment, which manifest themselves as diffusive effects. These phenomena will be discussed in our forthcoming publication.

\section{Discussion}

In the main part of this paper we have used a straightforward expansion in the quantum parameter $\hbar$. Such an expansion is, however, only formal, as no dimensionless small parameter has been extracted. From the nature of quantum theory, it follows that no universal combination of parameters characterizes the validity of the classical limit; the parameters of each separate problem have to be considered. In order to find a dimensionless validity condition, we look at the consecutive terms in the expression (3), where the convergence of the expansion requires that

$$
h^{\prime \prime} \frac{\partial^{n}}{\partial r^{n}} V \frac{\partial^{n}}{\partial p^{n}} W \ll V W
$$

We next introduce the formal expansion parameter

$$
\hbar \frac{\partial}{\partial r} \frac{\partial}{\partial p} \approx \frac{\hbar}{a \Delta p} \approx \frac{\lambda_{\mathrm{dB}}}{a},
$$

where $a$ is a length characterizing the potential $V(r)$ and $\Delta p$ is some momentum scale of the problem. For a nearly freely travelling particle, the momentum can change no faster than Planck's constant divided by the de Broglie wavelength $\lambda_{\mathrm{dB}}$. Thus the semiclassical expansion is good if $\lambda_{\mathrm{dB}} \ll a$.

In this work, we have assumed the wave function to be a localized wave packet of some finite size $\Delta r$ following the classical paths. We realize that the wave packet motion can represent classical dynamics only if its extension is much smaller than the characteristic dimensions of the potential where it moves. Combining this with (60) we require

$$
\lambda<\Delta r \ll a \text {. }
$$

Only in this limit can we find a situation where the classical trajectories can be defined, and we can construct localized wave functions that follow them. This is called the wave packet limit by Heller in Ref. [7]. 


\section{References}

[1] E.J. Heller, S. Tomsovic, Phys. Today 46, 38 (1993).

[2] M.S. Child, Semiclassical Mechanics with Molecular Applications, Clarendon Press, Oxford 1991.

[3] A.M. Ozorio de Almeida, IIamillonian Systems: Chaos and Quantization, Cambridge Univ. Press, Cambridge 1988.

[4] J.P. Dowling, W.P. Schlcich, J.A. Wheeler, Ann. Physik 7.48, 423 (1991).

[5] S. Stenholm, Phys. Rev. A 47, 2523 (1993).

[6] J. Kevorkian, J.D. Cole, Perturbation Methods in Applied Malhematics, Springer-Verlag, New York, 1981.

[7] E.J. Heller, J. Chem. Phys. 65, 1289 (1976).

[8] S. Stenholm, Rev. Mod. Phys. 58, 699 (1986).

[9] J.E. Moyal, Proc. Cambridge Phil. Soc. 45, 99 (1949).

[10] E.J. Heller, in: Chaos and Quanlum Physics, Leclures at the 1987 Les Ilouches Summer School, Eds. M.-J. Giannoni, A. Vovos, J. Zinn-Justin, Nortl-IIolland, Amsterdam 1991, p. 547. 